\title{
Dermatopathology Etiquette 101
}

\author{
Lauren Skudalski, BA; Ashley Elsensohn, MD, MPH; Amanda Kraus, BS; \\ Jacqueline M. Junkins-Hopkins, MD; Tammie C. Ferringer, MD; Eric Hossler, MD
}

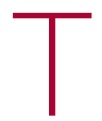
he Accreditation Council for Graduate Medical Education has established core competencies to serve as a foundation for the training received in a dermatology residency program. ${ }^{1}$ Although programs are required to have the same concentrations-patient care, medical knowledge, practice-based learning and improvement, interpersonal and communication skills, professionalism, and systems-based practice-no specific guidelines are in place regarding how each of these competencies should be reached within a training period. ${ }^{2}$ Instead, it remains the responsibility of each program to formulate an individualized curriculum to facilitate proficiency in the multiple areas encompassed by a residency.

In many dermatology residency programs, dermatopathology is a substantial component of educational objectives and the curriculum. ${ }^{1}$ Residents may spend as much as $25 \%$ of their training on dermatopathology. However, there is great variability among programs in methods of teaching dermatopathology. When Hinshaw ${ }^{3}$ surveyed 52 of 109 dermatology residency programs, they identified differences in dermatopathology teaching that included, but was not limited to, utilization of problembased learning (in $40.4 \%$ of programs), integration of journal reviews (53.8\%), and computer-based learning (19.2\%). In addition, differences were identified in the recommended primary textbook and the makeup of faculty who taught dermatopathology. ${ }^{3}$

Although residency programs vary in their methods of teaching this important component of dermatology, most use a multiheaded microscope in some capacity for didactics or sign-out. For most trainees, the dermatopathology laboratory is a new environment compared to the clinical space that medical students and residents become accustomed to throughout their education, thus creating a knowledge gap for trainees on proper dermatopathology etiquette and universal guidelines.

With medical students, residents, and fellows in mind, we have prepared a basic "dermatopathology etiquette" reference for trainees. Just as there are universal rules in the operating room for surgery (eg, sterile technique), we want to establish a code of conduct at the microscope. We hope that these 10 tips will, first, be useful to those who are unsure how to approach their first experience with dermatopathology and, second, serve as a guideline to aid development of appropriate communication skills and functioning within this novel setting. This list also can serve as a resource for dermatopathology attendings to provide to rotating residents and students.

1. New to pathology? It's okay to ask. Do not hesitate to ask upper-year residents, fellows, and attendings for instructions on such matters as how to adjust your eyepiece to get the best resolution.

2. If a slide drops on the floor, do not move! Your first instinct might be to move your chair to look for the dropped slide, but you might roll over it and break it.

3. When the attending is looking through the scope, you look through the scope. Dermatopathology is a visual exercise. Getting in your "optic mileage" is best done under the guidance of an experienced dermatopathologist.

4. Rules regarding food and drink at the microscope vary by pathologist. It's best to ask what each attending prefers. Safe advice is to avoid foods that make noise, such as chewing gum and chips, and food that has a strong odor, such as microwaved leftovers.

Ms. Skudalski is from Geisinger Commonwealth School of Medicine, Scranton, Pennsylvania. Dr. Elsensohn is from the University of California San Diego. Ms. Kraus is from Georgetown University School of Medicine, Washington, DC. Drs. Junkins-Hopkins, Ferringer, and Hossler are from Geisinger Medical Center, Danville, Pennsylvania.

The authors report no conflict of interest.

Correspondence: Lauren Skudalski, BA, Geisinger Commonwealth School of Medicine, 525 Pine St, Scranton, PA 18510 (Iskudalski@som.geisinger.edu).

doi:10.12788/cutis.0287 
5. Limit use of a laptop, cell phone, and smartwatch. If you think that using any of these is necessary, it generally is best to announce that you are looking up something related to the case and then share your findings (but not the most recent post on your Facebook News Feed).

6. If you notice that something needs correcting on the report, speak up! We are all human; we all make typos. Do not hesitate to mention this as soon as possible, especially before the case is signed out. You will likely be thanked by your attending because it is harder to rectify once the report has been signed out.

7. Small talk often is welcome during large excisions. This is a great time to ask what others are doing next weekend or what happened in clinic earlier that day, or just to tell a good (clean) joke that is making the rounds. Conversely, if the case is complex, it often is best to wait until it is completed before asking questions.

8. When participating in a roundtable diagnosis, you are welcome to directly state the diagnosis for bread-and-butter cases, such as basal cell carcinomas and seborrheic keratoses. It is appropriate to be more descriptive and methodical in more complex cases. When evaluating a rash, give the general inflammatory pattern first. For example, is it spongiotic? Psoriasiform? Interface? Or a mixed pattern?
9. Extra points for identifying special sites! These include mucosal, genital, and acral sites. You might even get bonus points if you can determine something about the patient (child or adult) based on the pathologic features, such as variation in collagen patterns.

10. Whenever you are in doubt, just describe what you see. You can use the traditional topdown approach or start with stating the most evident finding, then proceed to a top-down description. If it is a neoplasm, describe the overall architecture; then, what you see at a cellular level will get you some points as well.

We acknowledge that this list of 10 tips is not comprehensive and might vary by attending and each institution's distinctive training format. We are hopeful, however, that these 10 points of etiquette can serve as a guideline.

\section{REFERENCES}

1. Hinshaw M, Hsu P, Lee L-Y, et al. The current state of dermatopathology education: a survey of the Association of Professors of Dermatology. J Cutan Pathol. 2009;36:620-628. doi:10.1111/j.1600-0560.2008.01128.x

2. Hinshaw MA, Stratman EJ. Core competencies in dermatopathology. J Cutan Pathol. 2006;33:160-165. doi:10.1111/j.0303-6987.2006.00442.x

3. Hinshaw MA. Dermatopathology education: an update. Dermatol Clin. 2012;80:815-826. doi:10.1016/j.det.2012.06.003 